\title{
Efficacy of Anticholinergics for Chronic Prostatitis/Chronic Pelvic Pain Syndrome in Young and Middle-Aged Patients: A Single-Blinded, Prospective, Multi-Center Study
}

\author{
Doo Sang Kim, Yoon Soo Kyung ${ }^{1}$, Seung Hyo Woo ${ }^{2}$, Young Seop Chang ${ }^{3}$, Hyung-Jee Kim \\ Department of Urology, Soonchunhyang University College of Medicine, Cheonan; \\ ${ }^{1}$ Department of Urology, Dankook University College of Medicine, Cheonan; \\ ${ }^{2}$ Department of Urology, Eulji University School of Medicine, Daejeon; \\ ${ }^{3}$ Department of Urology, Konyang University College of Medicine, Daejeon, Korea
}

\begin{abstract}
Purpose: Chronic prostatitis/chronic pelvic pain syndrome (CP/CPPS) exhibits variable lower urinary tract symptoms (LUTS). The aim of this study was to evaluate the incidence of LUTS and the efficacy of an anticholinergic agent in young and middle-aged CP/CPPS patients.

Methods: Ninety-six men with CP/CPPS were randomly assigned in a single-blind fashion and received either ciprofloxacin (group 1, 49 patients) or ciprofloxacin and solifenacin (5 mg/day; group 2, 47 patients) for 8 weeks. The National Institutes of Health chronic prostatitis symptom index (NIH-CPSI), the International Prostate Symptom Score (IPSS), and the International Index of Erectile Function-5 (IIEF-5) were used to grade the patients' symptoms and the quality of life impact at the start of the study, and at 4 and 8 weeks from the initiation of the study.

Results: There was no significant difference between groups 1 and 2 with respect to age, duration of disease, or sub-domains of the IPSS, NIH-CPSI, or IIEF- 5 at baseline. Of these patients, $67.4 \%$ had LUTS. Statistically significant differences were determined via the NIH-CPSI for total score and the pain and urinary domain scores. Statistically significant differences were determined via the IPSS for total score and the storage domain score. The total score of the IIEF-5 increased, but the change was not significant. There was no statistically significant difference in residual urine.

Conclusions: Many CP/CPPS patients had LUTS. Solifenacin in CP/CPPS demonstrated improvements in the NIH-CPSI and the IPSS total score and storage score. Storage factors significantly improved via the NIH-CPSI and IPSS assessments in the solifenacin treatment group.
\end{abstract}

Keywords: Prostate; Cholinergic antagonists; Prostatitis

\section{INTRODUCTION}

Chronic prostatitis/chronic pelvic pain syndrome (CP/CPPS) is an enigma in urology although it is a common disorder [1]. Because it is diagnosed only on the basis of symptoms, principally pain or discomfort in the pelvic region [2], it may not be treated adequately. Although pain is the most severe and commonly reported symptom in patients with $\mathrm{CP} / \mathrm{CPPS}$, many patients have lower urinary tract symptoms (LUTS), especially storage symptoms, such as frequency and urgency [3-6].

Among the most commonly used treatments for CP/CPPS are antibiotics, $\alpha$-blockers, and anti-inflammatory drugs. Anticholinergic agents are used to treat frequency and urgency; however, the role of anticholinergics in the management of CP/CPPS is unknown, and there are few references on the topic [7].

The aim of this study was to evaluate the prevalence of LUTS
Corresponding author: Hyung-Jee Kim

Department of Urology, Dankook University College of Medicine, San 29 Anseo-dong, Dongnam-gu, Cheonan 330-715, Korea

Tel: +82-41-550-6630 / Fax: +82-41-556-0524 / E-mail : killtumor@yahoo.co.kr Submitted: March 16, 2011 / Accepted after revision: August 24, 2011
This is an Open Access article distributed under the terms of the Creative Commons Attribution Non-Commercial License (http://creativecommons.org/licenses/by-nc/3.0/) which permits unrestricted non-commercial use, distribution, and reproduction in any medium, provided the original work is properly cited. 
and the efficacy of solifenacin in a prospective, randomized, single-blinded, multi-centered clinical trial of young and middle-aged CP/CPPS patients.

\section{MATERIALS AND METHODS}

This study was performed under the Institutional Review Board approval of each clinical trial center, and written informed consent was given by all patients. At four Korean centers, a total of 96 patients with CP/CPPS were randomly assigned into group 1 ( $\mathrm{n}=49$; ciprofloxacin $1,000 \mathrm{mg}$ once daily) or group $2(\mathrm{n}=47$; ciprofloxacin 1,000 $\mathrm{mg}$ and solifenacin $5 \mathrm{mg}$ once daily) with single blinded randomization method. All patients had a urine analysis, urine culture, expressed prostatic secretions examination, prostate-specific antigen (PSA) measurement, uroflowmetry, and postvoid residual volume (PVR) measurement. The International Prostate Symptom Score (IPSS), the National Institutes of Health Chronic Prostatitis Symptom Index (NIH-CPSI), and the International Index of Erectile Function-5 (IIEF-5) were used to grade the patients' symptoms and the quality of life (QoL) impact at the starting point (baseline) and at 4 and 8 weeks into the study.

Patients aged 20 to 49 years were included to exclude the LUTS of benign prostatic hyperplasia (BPH). Other inclusion criteria were patients diagnosed with CP/CPPS (NIH-CPSI category IIIa and IIIb) and with pelvic pain for 3 or more months, negative urine culture and expressed prostatic secretion results, PVR of $100 \mathrm{~mL}$ or less, maximum urinary flow rate (Qmax) of $15 \mathrm{~mL} / \mathrm{sec}$ or greater, a total NIH-CPSI score of 8 or greater, and an NIH-CPSI pain score of 4 or greater at baseline. Exclusion criteria included a history of previous prostate surgery, $5 a$-reductase inhibitor use for 3 or more months, anticholinergic use within 6 months of baseline, PSA level of $4.0 \mathrm{ng} / \mathrm{mL}$ or more, prostatic cancer, urethral stricture, diabetes mellitus, neurogenic bladder and hypersensitivity to ciprofloxacin $(1,000$ $\mathrm{mg}$ ) or solifenacin.

Statistical analysis was performed by repeated-measures analysis of variance with SPSS ver. 12.0 (SPSS Inc., Chicago, IL, USA), and $\mathrm{P}<0.05$ was considered to indicate significance.

\section{RESULTS}

There was no significant difference between group 1 and 2 at the baseline when the age, duration of disease, PVR, Qmax, scores on the IPSS, NIH-CPSI or IIEF-5 were compared. Among those patients, $67.4 \%$ demonstrated LUTS (IPSS $\geq 8$ ). In NIH-CPSI score, there were statistically significant differences between group I and II in total ( $12.1 \pm 7.4$ vs. $10.8 \pm 5.4, \mathrm{P}=0.01)$, pain $(5.3 \pm 3.6$ vs. $13.9 \pm 5.0, \mathrm{P}=0.04)$ and urinary domain scores $(1.9 \pm 1.6$ vs. $2.1 \pm 1.8, \mathrm{P}=0.01)$ at 8 weeks of treatment. Whereas after 8 weeks of treatment, there was no statistical differences in QoL scores between group I and II: $5.1 \pm 3.0$ vs. $5.1 \pm 1.8$, $\mathrm{P}=0.13$ (Table 1). Statistically significant differences were found in the IPSS total score and storage domain score between group I and II at 8 weeks of treatment. IPSS total score was $5.3 \pm 5.5$ and $7.2 \pm 4.7(\mathrm{P}=0.04)$, and the storage was $2.0 \pm 2.2$ and $3.2 \pm 2.3(\mathrm{P}=0.01)$, respectively. Also, these scores were significantly decreased compared to the baseline scores. Even though, voiding scores ( $3.4 \pm 3.7$ vs. $4.0 \pm 3.2, \mathrm{P}=0.22)$ and $\mathrm{QoL}$ scores $(1.7 \pm 1.2$ vs. $2.4 \pm 1.4, \mathrm{P}=0.38)$ were decreased from the mean scores during 8 weeks of treatment, there was no statistical differences (Table 2). The total score of the IIEF-5 was increased without any significant statistical differences (Table 3).

\section{DISCUSSION}

$\mathrm{CP} / \mathrm{CPPS}$ is a common disorder in men, and the syndrome is diagnosed only on the basis of symptoms, principally pain or discomfort in the pelvic region. Because the pathophysiology of

Table 1. Comparison of the NIH-CPSI results in the pre-treatment and post-treatment groups

\begin{tabular}{lrrr} 
& \multicolumn{3}{c}{ NIH-CPSI } \\
\cline { 2 - 4 } & Baseline & $4 \mathrm{wk}$ & $8 \mathrm{wk}$ \\
\hline Group 1 $(\mathrm{n}=40)$ & & & \\
Total & $19.8 \pm 6.4$ & $14.5 \pm 7.6$ & $12.1 \pm 7.4$ \\
Pain & $9.3 \pm 3.2$ & $6.5 \pm 3.9$ & $5.3 \pm 3.6$ \\
Urinary & $2.9 \pm 2.2$ & $2.2 \pm 1.9$ & $1.9 \pm 1.6$ \\
QoL & $7.8 \pm 3.0$ & $6.1 \pm 3.3$ & $5.1 \pm 3.3$ \\
Group 2 $(\mathrm{n}=47)$ & & & \\
Total & $22.4 \pm 6.0$ & $13.9 \pm 5.0$ & $10.8 \pm 5.4^{\mathrm{a})}$ \\
Pain & $9.4 \pm 3.3$ & $5.1 \pm 3.0$ & $3.9 \pm 2.9^{\mathrm{a})}$ \\
Urinary & $4.9 \pm 2.6$ & $2.8 \pm 2.2$ & $2.1 \pm 1.8^{\mathrm{a})}$ \\
QoL & $8.4 \pm 2.7$ & $6.1 \pm 2.6$ & $5.1 \pm 1.8$ \\
\hline
\end{tabular}

Values are presented as mean $\pm \mathrm{SD}$.

NIH-CPSI, National Institutes of Health chronic prostatitis symptom index; QoL, quality of life.

Group 1: antibiotics alone. Group 2: antibiotics and solifenacin $(5 \mathrm{mg})$.

a) Indicates a significant difference from group $1(\mathrm{P}<0.05)$ by repeated measure analysis of variance. 
Table 2. Comparison of the IPSS results in the pre-treatment and post-treatment groups

\begin{tabular}{lccc}
\hline & \multicolumn{3}{c}{ NIH-CPSI } \\
\cline { 2 - 4 } & Baseline & $4 \mathrm{wk}$ & $8 \mathrm{wk}$ \\
\hline Group 1 $(\mathrm{n}=40)$ & & & \\
$\quad$ Total & $9.0 \pm 8.3$ & $6.6 \pm 5.2$ & $5.3 \pm 5.5$ \\
Storage & $3.3 \pm 3.2$ & $2.6 \pm 2.5$ & $2.0 \pm 2.2$ \\
Voiding & $5.7 \pm 5.5$ & $4.0 \pm 4.2$ & $3.4 \pm 3.7$ \\
QoL & $2.7 \pm 1.6$ & $2.1 \pm 1.5$ & $1.7 \pm 1.2$ \\
Group 2 $(\mathrm{n}=47)$ & & & \\
Total & $13.5 \pm 7.1$ & $9.0 \pm 5.3$ & $7.2 \pm 4.7^{\mathrm{a})}$ \\
Storage & $5.9 \pm 2.7$ & $4.0 \pm 2.5$ & $3.2 \pm 2.3^{\mathrm{a})}$ \\
Voiding & $7.5 \pm 5.0$ & $5.0 \pm 3.9$ & $4.0 \pm 3.2$ \\
QoL & $3.5 \pm 1.6$ & $2.9 \pm 1.5$ & $2.4 \pm 1.4$ \\
\hline
\end{tabular}

Values are presented as mean \pm SD.

IPSS, International Prostate Symptom Score; QoL, quality of life. Group 1: antibiotics alone. Group 2: antibiotics and solifenacin $(5 \mathrm{mg})$. ${ }^{a}$ Indicates a significant difference from group $1(\mathrm{P}<0.05)$ by repeated measure analysis of variance.

the disease has not been established, antimicrobial agents are frequently used. However a patient who does not respond to antibiotics might need additional treatments, such as nonsteroidal anti-inflammatory drugs, alpha-blocking agents, or other therapies for symptomatic relief $[1,8]$.

Dmochowski and Gomelsky [9] suggested that CP/CPPS was another condition with prominent LUTS. The condition is typically accompanied by pain with ejaculation or in the perineal area. Because there is no gold standard diagnostic test for $\mathrm{CP} /$ CPPS and because its etiology is largely unknown, this entity remains a diagnosis of exclusion. As with $\mathrm{BPH}$, the symptoms of CP/CPPS can be present during the storage phase, can be present during the voiding phase, or can occur postmicturition [9].

A recent study showed that because CP/CPPS is an important multifactorial problem that affects men of all ages and demographic subgroups, patients with CP/CPPS receive many empirical therapies. The three most common treatments prescribed by physicians are antimicrobial agents, anti-inflammatory medicines, and alpha-adrenergic receptor antagonists [10]. Many theories exist on the efficacy of the medicines prescribed; however, there remains a lack of scientific evidence to prove their efficacy [11]. Therefore, there is a need for a long-term, randomized, controlled study to evaluate the efficacy of treatment, especially because controversy concerning the efficacy and mechanism of action of alpha-blockers in the treatment of CP/CPPS.
Table 3. Comparison of the IIEF results in the pre-treatment and post-treatment groups

\begin{tabular}{lcccc}
\hline & & \multicolumn{3}{c}{ NIH-CPSI } \\
\cline { 3 - 5 } & & Baseline & $4 \mathrm{wk}$ & $8 \mathrm{wk}$ \\
\hline Group 1 $(\mathrm{n}=40)$ & Total & $17.3 \pm 5.2$ & $17.6 \pm 5.6$ & $17.2 \pm 5.8$ \\
Group 2 $(\mathrm{n}=47)$ & Total & $17.8 \pm 4.9$ & $18.1 \pm 5.6$ & $18.2 \pm 5.9$ \\
\hline
\end{tabular}

Values are presented as mean \pm SD.

IIEF-5, International Index of Erectile Function-5.

Group 1: antibiotics alone. Group 2: antibiotics and solifenacin (5 mg).

Furthermore, many studies have investigated the efficacy of antimicrobial agents and alpha-adrenergic receptor antagonists, but there have been few studies of the efficacy of other medicines used in empirical treatment, such as plant extracts, zinc, antidepressants, anti-anxiety medications, 5-alpha reductase inhibitors, narcotics, analgesics, anticholinergics, or antispasmodics.

In recent reports, the primary symptoms of $\mathrm{CP} / \mathrm{CPPS}$ were pelvic pain and frequent urination $[4,6,12]$.

However, many physicians have used anticholinergics empirically for treatment, and there are only introductions to treatment with anticholinergics in some of the guidelines [7].

In this study, to evaluate the prevalence of LUTS and the efficacy of anticholinergics, we used the IPSS, NIH-CPSI, and IIEF5 to grade the symptoms and QoL impact. In our study, only young and middle-aged patients were included to exclude the LUTS of BPH because $\mathrm{BPH}$ patients have a higher prevalence of LUTS than do healthy men and a higher prevalence of sexrelated symptoms [13]. In the present study, we found statistically significant differences between the two groups in the total score, pain domain, and urinary domain of the NIH-CPSI and the total score and storage symptom score of the IPSS. In a recent study investigating treatment of CP/CPPS with alfuzosin, alfuzosin improved only the voiding symptom score determined via the IPSS and not the storage symptoms assessed by the IPSS or any of the domains in the NIH-CPSI [14], Such finding provide the basis for believing that combined therapy with alphablockers and anticholinergics may show synergistic effects in the management of $\mathrm{CP} / \mathrm{CPPS}$. The IPSS assessment appears to be a good indicator for follow-up in the management of CP/ CPPS, especially in patients with severe LUTS [12]. Solifenacin improved the pain associated with CP/CPPS in our study, but we think that this was probably because some patients with interstitial cystitis were included in the study.

Our data suggests that anticholinergics are effective in the management of $\mathrm{CP} / \mathrm{CPPS}$, especially for the treatment of storage 
symptoms. However, because the number of patients was small and the period of the clinical trial was short, more randomized, controlled, long-term and large-scale clinical trials are needed.

In our study, the scores between the two groups were compared. However, to prove the effective of anticholinergics in $\mathrm{CP} /$ CPPS, absolute decreased values between two groups should be considered during the study.

In conclusion, many CP/CPPS patients had LUTS. Solifenacin demonstrated efficancy in CP/CPPS in improving the NIHCPSI and the IPSS total score and storage score. Storage factors improved significantly, as determined via the NIH-CPSI and IPSS in the solifenacin treatment group.

\section{CONFLICT OF INTEREST}

No potential conflict of interest relevant to this article was reported.

\section{REFERENCES}

1. Collins MM, Meigs JB, Barry MJ, Walker Corkery E, Giovannucci E, Kawachi I. Prevalence and correlates of prostatitis in the health professionals follow-up study cohort. J Urol 2002;167:1363-6.

2. Krieger JN, Nyberg L Jr, Nickel JC. NIH consensus definition and classification of prostatitis. JAMA 1999;282:236-7.

3. Luzzi GA. Chronic prostatitis and chronic pelvic pain in men: aetiology, diagnosis and management. J Eur Acad Dermatol Venereol 2002;16:253-6.

4. Liang CZ, Zhang XJ, Hao ZY, Yang S, Wang DB, Shi HQ, et al. An epidemiological study of patients with chronic prostatitis. BJU Int 2004;94:568-70.
5. Schaeffer AJ. Epidemiology and demographics of prostatitis. Eur urol suppl 2003;2:5-10.

6. Clemens JQ, Nadler RB, Schaeffer AJ, Belani J, Albaugh J, Bushman W. Biofeedback, pelvic floor re-education, and bladder training for male chronic pelvic pain syndrome. Urology 2000;56:951-5.

7. Fall M, Baranowski AP, Fowler CJ, Lepinard V, Malone-Lee JG, Messelink EJ, et al. EAU guidelines on chronic pelvic pain. Eur Urol 2004;46:681-9.

8. Collins MM, Stafford RS, O'Leary MP, Barry MJ. How common is prostatitis? A national survey of physician visits. J Urol 1998;159: 1224-8.

9. Dmochowski RR, Gomelsky A. Overactive bladder in males. Ther Adv Urol 2009;1:209-21.

10. Schaeffer AJ, Landis JR, Knauss JS, Propert KJ, Alexander RB, Litwin MS, et al. Demographic and clinical characteristics of men with chronic prostatitis: the national institutes of health chronic prostatitis cohort study. J Urol 2002;168:593-8.

11. McNaughton Collins M, MacDonald R, Wilt TJ. Diagnosis and treatment of chronic abacterial prostatitis: a systematic review. Ann Intern Med 2000;133:367-81.

12. Litwin MS. A review of the development and validation of the National Institutes of Health Chronic Prostatitis Symptom Index. Urology 2002;60(6 Suppl):14-8.

13. Rosen R, Altwein J, Boyle P, Kirby RS, Lukacs B, Meuleman E, et al. Lower urinary tract symptoms and male sexual dysfunction: the multinational survey of the aging male (MSAM-7). Eur Urol 2003; 44:637-49.

14. Ryu YG, Kim HJ, Park HJ. The efficacy of alfuzosin for chronic prostatitis/chronic pelvic pain syndrome in young and middle aged patients. Korean J Urol 2007;48:858-62. 